\title{
Raquianestesia com Morfina Versus Raquianestesia sem Morfina Associada a Bloqueio do Nervo Pudendo. Avaliação da Analgesia e Complicações em Hemorroidectomias
}

\author{
Spinal Anesthesia with Morphine Versus Spinal Anesthesia Without Morphine \\ Associated to Pudendal Nerve Block. Evaluation of the Analgesia and \\ Complications in Hemorrhoidectomy
}

${ }^{1}$ CARLOS HENRIQUE MARQUES DOS SANTOS; ${ }^{2}$ FÁBIO TACLA SAAD; ${ }^{3}$ ROBSON LUIZ SILVEIRA JARÁ;
${ }^{4}$ MAGALIDA SILVA SANCHES MACHADO.

\begin{abstract}
${ }^{1}$ Mestre e Doutor em Medicina, Titular da Sociedade Brasileira de Coloproctologia, Titular do Colégio Brasileiro de Cirurgiões, Cirurgião Geral do Hospital Regional de Mato Grosso do Sul; ${ }^{2}$ Residente de Cirurgia Geral do Hospital Regional de Mato Grosso do Sul; ${ }^{3}$ Cirurgião Geral do Hospital Regional de Mato Grosso do Sul, Titular do Colégio Brasileiro de Cirurgia Digestiva; ${ }^{4}$ Mestre e Doutora em Medicina, Cirurgiã Geral do Hospital Regional de Mato Grosso do Sul.
\end{abstract}

\begin{abstract}
SANTOS CHM; SAAD FT; JARA RLS; MACHADO MSS. Raquianestesia com Morfina Versus Raquianestesia sem Morfina Associada a Bloqueio do Nervo Pudendo. Avaliação da Analgesia e Complicações em Hemorroidectomias. Rev bras Coloproct, 2009;29(1): 046-050.

RESUMO: INTRODUÇÃO: Recentemente, alguns autores têm relatado que o bloqueio do nervo pudendo com uso de ropivacaína proporciona boa analgesia pós-operatória. Assim, considerou-se de interesse a comparação de dois métodos anestésicos verificando a analgesia pós-operatória e as complicações. OBJETIVO: Comparar duas técnicas anestésicas (raquianestesia com morfina versus raquianestesia sem morfina associada com bloqueio do nervo pudendo) para hemorroidectomia quanto à analgesia pósoperatória e complicações. MATERIAIS E MÉTODOS: Foram estudados 40 pacientes provenientes do ambulatório de Coloproctologia do Hospital Regional de Mato Grosso do Sul no período de março a outubro de 2008. Todos os pacientes foram randomizados por sorteio imediatamente antes de se iniciar a intervenção cirúrgica e foram distribuídos em dois grupos: Grupo 1: 20 pacientes submetidos à raquianestesia com morfina com 0,15 $\mu$ g associado com bupivacaína hiperbárica 10mg; - Grupo 2: 20 pacientes submetidos à raquianestesia com bupivacaína pesada $10 \mathrm{mg}$ associado ao bloqueio do nervo pudendo bilateral com agulha para raquianestesia $27 \mathrm{G}$ utilizando ropivacaína $10 \mathrm{mg} / \mathrm{ml}$ diluída $\mathrm{em} 10 \mathrm{ml}$ de água destilada. RESULTADOS: Avaliou-se 40 pacientes, sendo 21 do sexo feminino. A idade média foi de 52 anos, variando de 21 a 72 anos. Apenas no grupo 1 observou-se complicações, sendo a principal prurido, seguida de retenção urinária e cefaléia. Após 6 horas da cirurgia, a média do nível de dor foi 1,55 no grupo 1 e 1,4 no grupo 2. Após 12 horas da cirurgia as médias foram 1,75 e 0,15 nos grupos 1 e 2, respectivamente. CONCLUSÃO: A hemorroidectomia realizada com associação de raquianestesia sem morfina e bloqueio do nervo pudendo utilizando ropivacaína proporcionou melhor efeito analgésico no período de 12 horas após a cirurgia e não apresentou complicações nesta casuística, mostrando-se superior à raquianestesia com morfina.
\end{abstract}

Descritores: Hemorroidas, cirurgia, raquianestesia, complicações.

\section{INTRODUÇÃO}

A doença hemorroidária acomete $4,4 \%$ da população geral nos Estados Unidos, podendo ocorrer em ambos os sexos ${ }^{1}$. Entre todas as modalidades do trata- mento da doença hemorroidária, a ressecção cirúrgica parece ser a que melhor elimina os sintomas e proporciona uma melhor qualidade de vida aos pacientes ${ }^{2-3}$.

Levando em consideração que o pós-operatório da hemorroidectomia é marcado pela dor,

Trabalho realizado no Serviço de Cirurgia Geral do Hospital Regional de Mato Grosso do Sul.

Recebido em 30/01/2009

Aceito para publicação em 14/03/2009 
muitas vezes intensa, fato que inibe os pacientes de procurar tratamento, muitos fatores tem sido analisados visando minimizar este sintoma. Dentre eles a técnica (aberta, fechada, etc.), as diferentes fontes de energia (bisturi frio, eletrocirurgia mono ou bipolar, etc.) e a técnica anestésica utilizada $^{4}$.

O alivio da dor pós-operatória, além de proporcionar maior conforto ao doente, pode também diminuir o tempo de internação, os custos hospitalares e pode proporcionar o retorno mais precoce às atividades habituais ${ }^{5}$.

Diversos métodos analgésicos têm sido preconizados para alívio da dor pós-operatória em hemorroidectomias como a morfina subcutânea com bomba de infusão, a estimulação elétrica transcutânea, uso de corticoides, a infiltração perineal com bupivacaína, bloqueio posterior do períneo e o bloqueio da fossa isquiorretal, sem que, no entanto, tenham efetivamente resolvido a questão com baixo custo e fácil aplicabilidade ${ }^{2}$.

A hemorroidectomia é realizada na maioria das vezes com raquianestesia ou anestesia peridural, anestesia local com ou sem sedação e raramente com anestesia geral. A raquianestesia com associação de bupivacaína e morfina proporciona uma excelente analgesia, sendo por isso bastante utilizada. Entretanto, tem como um de seus maiores inconvenientes a retenção urinária que pode chegar a até $20 \%$, postergando a alta destes enfermos e causando-lhes grande desconforto ${ }^{6}$.

Recentemente, alguns autores têm relatado que o bloqueio do nervo pudendo com uso de ropivacaína proporciona boa analgesia pós-operatória $^{6}$. Assim, consideramos de interesse a comparação de um método anestésico tradicional (raquianestesia com morfina), mas que apresenta efeitos indesejados, com a raquianestesia sem morfina associada ao bloqueio do nervo pudendo com ropivacaína, com objetivo de comparar a analgesia pós-operatória e as complicações.

\section{OBJETIVO}

Comparar duas técnicas anestésicas (raquianestesia com morfina versus raquianestesia sem morfina associada com bloqueio do nervo pudendo) para hemorroidectomia quanto à analgesia e complicações pós-operatórias.

\section{MATERIAIS E MÉTODOS}

O estudo foi aprovado pela Comissão de Ética do Hospital Regional de Mato Grosso do Sul, mediante informação completa sobre o estudo para o paciente e assinatura do Termo de Consentimento Livre e Esclarecido. Não houve conflito de interesses.

Critérios de inclusão: idade maior de 13 anos e hemorroidas de graus III ou IV.

Critérios de exclusão: idade menor de 12 anos, hemorroidas de grau I e II, hemorroidas de grau III e IV associadas a outras doenças anais (fístulas, fissuras, etc).

Foram estudados 40 pacientes provenientes do ambulatório de Coloproctologia do Hospital Regional de Mato Grosso do Sul no período de março a outubro de 2008. A idade variou de 21 a 72 anos, com média de 52 anos. Foi administrado Cefoxitina $2 \mathrm{~g}$ IV na indução anestésica em todos pacientes. Os pacientes foram randomizados por sorteio imediatamente antes de se iniciar a intervenção cirúrgica, sendo distribuídos em dois grupos:

- Grupo 1: 20 pacientes submetidos à raquianestesia com morfina $0,15 \mu$ g associado com bupivacaína hiperbárica $10 \mathrm{mg}$, utilizando agulha para raquianestesia $27 \mathrm{G}$, sendo a punção realizada entre $\mathrm{L} 3$ e L4, com o paciente sentado.

- Grupo 2: 20 pacientes submetidos à raquianestesia com bupivacaína pesada $10 \mathrm{mg}$, utilizando agulha para raquianestesia $27 \mathrm{G}$, sendo a punção realizada entre L3 e L4, com o paciente sentado. Após a perda da sensibilidade perineal, com o paciente em posição de litotomia, realizou-se o bloqueio do nervo pudendo interno bilateral com agulha para raquianestesia $27 \mathrm{G}$ introduzida em raiz da coxa posterior em direção à espinha isquiática utilizando ropivacaína $10 \mathrm{mg} / \mathrm{ml}$ diluída em $10 \mathrm{ml}$ de água destilada, instilando-se $10 \mathrm{ml}$ de cada lado.

A hemorroidectomia foi realizada por técnica fechada com poliglactina 4-0 em todos os pacientes. Utilizou-se no pós-operatório Dipirona $2 \mathrm{ml}$ IV a cada 6 horas e Cetoprofeno 100mg IV a cada 12 horas para analgesia. Em caso de retenção urinária realizava-se sondagem vesical de alívio.

Os resultados foram catalogados no período de internação do paciente onde foram analisadas as seguintes variáveis:

1. Dor pós-operatória ( $6 \mathrm{~h}$ e $12 \mathrm{~h}$ seguintes ao procedimento), utilizando escala de dor de 0 a 10 , de 
Raquianestesia com Morfina Versus Raquianestesia sem Morfina Associada a Bloqueio do Nervo Pudendo. Avaliação da Analgesia e Complicações em Hemorroidectomias

Carlos Henrique Marques dos Santos e Cols.
Vol. 29 acordo com a intensidade da dor, considerando-se 0 a ausência completa de dor e 10 dor de extrema intensidade.

2. Complicações, onde se avaliou: prurido, náuseas, vômito, cefaleia e retenção urinária.

Os resultados foram submetidos a tratamento estatístico pelo teste $t$ de Student, considerando-se significativo $\mathrm{p}<0,05$.

\section{RESULTADOS}

No primeiro momento de avaliação pós-operatória (6h) o nível de dor no grupo 1 variou de 0 a 4 , com média 1,55. No grupo 2 houve variação de 0 a 7 , com média 1,4 (tabela 1), demonstrando não haver diferença entre os grupos $(\mathrm{p}=0,811)$.

Após 12 horas da cirurgia, os pacientes do grupo 1 apresentaram graus de dor variando de 0 a 5 , com média 1,75 , enquanto que os do grupo 2 tiveram varia-

Tabela 1 - Avaliação do nível de dor após 6 horas da cirurgia nos grupos 1 e 2.

\begin{tabular}{lcc}
\hline Pacientes & \multicolumn{2}{c}{ Nível de dor } \\
\cline { 2 - 3 } & Grupo 1 & Grupo 2 \\
\hline 1 & 2 & 4 \\
2 & 3 & 2 \\
3 & 0 & 4 \\
4 & 0 & 0 \\
5 & 0 & 5 \\
6 & 4 & 0 \\
7 & 0 & 0 \\
8 & 0 & 0 \\
9 & 0 & 0 \\
10 & 1 & 0 \\
11 & 3 & 0 \\
12 & 2 & 0 \\
13 & 0 & 0 \\
14 & 3 & 0 \\
15 & 2 & 0 \\
16 & 1 & 0 \\
17 & 4 & 2 \\
18 & 3 & 2 \\
19 & 2 & 0 \\
20 & 1 & 7 \\
Média & 1,55 & 1,4 \\
\hline
\end{tabular}

$p=0,811$. ção de 0 a 2, com média 0,15 (tabela 2), demonstrando efeito analgésico mais prolongado no grupo $2 \mathrm{com}$ diferença estatísticamente significativa $(\mathrm{p}=0,0008)$.

Os pacientes do grupo 2 não apresentaram complicações, enquanto que no grupo 1 observou-se 25 complicações, sendo que alguns destes pacientes apresentaram mais de uma. A principal complicação observada foi o prurido (12) seguido por retenção urinária (9). Não houve complicações graves nem óbitos nesta casuística (tabela 3). Pode-se constatar que o grupo 2 apresentou vantagem em relação ao grupo 1 quanto a presença de prurido e retenção urinária $(\mathrm{p}<0,0001)$.

\section{DISCUSSÃO}

A raquianestesia pode causar distúrbios da função vesical devido à interrupção do reflexo da micção, especialmente nas hemorroidectomias, com incidência

Tabela 2 - Avaliação do nível de dor após 12 horas da cirurgia nos grupos 1 e 2.

\begin{tabular}{lcc}
\hline Pacientes & \multicolumn{2}{c}{ Nível de dor } \\
\cline { 2 - 3 } & Grupo 1 & Grupo 2 \\
\hline 1 & 2 & 0 \\
2 & 2 & 0 \\
3 & 0 & 0 \\
4 & 0 & 0 \\
5 & 0 & 2 \\
6 & 5 & 0 \\
7 & 0 & 0 \\
8 & 0 & 0 \\
9 & 0 & 0 \\
10 & 1 & 0 \\
11 & 3 & 0 \\
12 & 2 & 0 \\
13 & 3 & 0 \\
14 & 4 & 0 \\
15 & 3 & 0 \\
16 & 3 & 0 \\
17 & 2 & 0 \\
18 & 2 & 1 \\
19 & 2 & 0 \\
20 & 1 & 0 \\
Média & 1,75 & 0,15 \\
\hline
\end{tabular}

$p=0,0008$. 
Tabela 3 - Complicações apresentadas no período pós-operatório nos pacientes dos grupos 1 e 2.

\begin{tabular}{lcc}
\hline Complicação & Número de pacientes que apresentaram complicação(ões) \\
\cline { 2 - 3 } & Grupo 1 & Grupo 2 \\
\hline Prurido & 12 & $0^{*}$ \\
Cefaleia & 3 & 0 \\
Náusea & 0 & 0 \\
Vômito & 1 & 0 \\
Retenção urinária & 9 & $0^{*}$ \\
Total & 25 & 0 \\
\hline
\end{tabular}

${ }^{*} p<0,0001$.

média de $15 \%$ variando de $1 \%$ a $52 \%$ dos pacientes $^{7-11}$. A retenção urinária provavelmente está relacionada à disfunção do músculo detrussor ou espasmo uretral secundário à dor e bloqueio remanescente do parassimpático sacral, onde a função da bexiga permanece imperfeita até que o bloqueio tenha regredido ao $3^{\circ}$ segmento sacral ${ }^{10-12}$. No presente estudo utilizou-se morfina associada com bupivacaína hiperbárica, observando-se retenção urinária em 22,5\% dos pacientes do grupo 1, o que não ocorreu no grupo 2. Este fato provavelmente se deve a não utilização da morfina no grupo 2 , medicamento que habitualmente causa este tipo de complicação, demonstrando assim um benefício na técnica anestésica utilizada no grupo 2 .

A associação morfina e bupivacaína hiperbárica utilizada no grupo 1 favoreceu a ocorrência de prurido generalizado, com incidência de $30 \%$, porém de fácil controle com administração de anti-histamínicos, conforme exposto na literatura ${ }^{13-}$ 14. Também esta complicação pode ser decorrente do emprego da morfina, já que não foi observada no grupo 2.

Verificou-se no presente estudo uma incidência de 7,5\% quanto à ocorrência de cefaleia pós raquianestesia, bem inferior a encontrada na literatura, podendo estar associado com o número de punções para se atingir o espaço e o calibre da agulha para a punção. Nesta pesquisa, foram utilizadas agulhas de calibre $27 \mathrm{G}$, o que diminui o índice desse efeito colateral ${ }^{15}$.

Em seu estudo Imbelloni e col. ${ }^{15}$ verificaram que utilizando uma dose mínima de morfina para realizar a raquianestesia pode-se obter diversas vantagens, particularmente para cirurgias ambulatoriais como recuperação rápida, estabilidade hemodinâmica, risco reduzido de sintomas neurológicos temporários, baixa incidência de retenção urinária e facilitação de locomoção precoce. Este e outros estudos têm sido realizados com o objetivo de se diminuir as complicações da raquianestesia com morfina nas cirurgias proctológicas, uma excelente técnica anestésica do ponto de vista analgésico, mas que apresenta reações extremamente incômodas.

Acredita-se que o presente estudo demonstrou uma boa opção anestésica para tais procedimentos, uma vez que o controle da dor no grupo 2 foi semelhante ou melhor à técnica padrão-ouro e com a vantagem de não apresentar complicações.

\section{CONCLUSÃO}

A hemorroidectomia realizada com associação de raquianestesia sem morfina e bloqueio do nervo pudendo utilizando ropivacaína proporcionou melhor efeito analgésico no período de 12 horas após a cirurgia e não apresentou complicações nesta casuística, mostrando-se superior à raquianestesia com morfina. 
Rev bras Coloproct Janeiro/Março, 2009
Raquianestesia com Morfina Versus Raquianestesia sem Morfina Associada a Bloqueio do Nervo Pudendo. Avaliação da Analgesia e Complicações em Hemorroidectomias Carlos Henrique Marques dos Santos e Cols.

\begin{abstract}
INTRODUCTION: Recently, some authors have reported that the pudendal nerve block using ropivacaine provides good postoperative analgesia. Thus, we consider of interest to compare two anesthetic methods checking postoperative analgesia and adverse effects. OBJECTIVE: To compare two anesthetic techniques (spinal anesthesia with morphine versus spinal anesthesia without morphine associated with pudendal nerve block) for hemorrhoidectomy in relation to postoperative analgesia and adverse effects. MATERIALS AND METHODS: We studied 40 patients from Hospital Regional de Mato Grosso do Sul among March to October of 2008. All patients were randomized by lot immediately before surgery, distributed in two groups: Group 1: 20 patients undergoing spinal anesthesia with morphine $0.15 \mu$ associated with hyperbaric bupivacaine 10mg; - Group 2: 20 patients undergoing spinal anesthesia with heavy bupivacaine 10mg associated with bilateral pudendal nerve block with ropivacaine $10 \mathrm{mg} / \mathrm{ml}$ diluted in $10 \mathrm{ml}$ of distilled water. RESULTS: Most patients were female $(\mathrm{N}=21)$. The average age was 52 years, ranging from 21 to 72 years. The group 1 presented a higher prevalence of adverse effects $(62.5 \%)$. Among the adverse effects of group 1 was higher prevalence of itch, urinary retention and migraine. Most patients who reported pain in the postoperative range from group 1 was male and prevailing in the first 12 hours, with the average level of pain of 1.55. In group 2 the pain was observed in equal proportion in both sexes and in the first six hours, with the average level of pain of 1.75. CONCLUSION: The hemorrhoidectomy accomplished with spinal anesthesia without morphine and blockade of the pudendal nerve using ropivacaine provided better analgesic effect in the period of 12 hours after the surgery and it didn't present complications in this casuistry, being shown superior to the spinal anesthesia with morphine.
\end{abstract}

Key words: Hemorrhoids, surgery, spinal anesthesia, complications.

\section{REFERÊNCIAS}

1. Moreira JPT, Araújo SEA, Oliveira JR O. Diagnóstico da hemorróida, Projeto Diretrizes. Rev Assoc Med Bras 2007; 53(1): 10-11.

2. Imbelloni LE, Beato L, Beato C Cordeiro JA, Souza DD. Analgesia pós-operatória com bloqueio bilateral do nervo pudendo com bupivacaína S75:R25 a 0,25\%. Estudo piloto em hemorroidectomia sob regime ambulatorial. Rev Bras Anestesiol 2005; 55(6): 614-21.

3. Nelson H. Ânus. In: Towsend, editor. Sabiston Tratado de Cirurgia. 17.a ed. Rio de Janeiro: Editora Saunders Elsevier 2005;49: 1490-1.

4. Moreira JPT, Araújo SEA, Oliveira Jr O. Hemorróidas: Tratamento Cirúrgico. Rev Bras Coloproctol 2006; 2(16):1-10.

5. Lacerda Filho A, Cunha MJR. Hemorroidectomia em regime ambulatorial sob anestesia local - Estudo prospectivo de 50 casos. Rev Bras Coloproct 1995; 15 (4): 206-10.

6. Gabrielli F, Cioffi V, Chiarelli M, Guttadauro A, Simone M. Hemorrhoidectomy with posterior perineal block. Dis Colon Rectum 2000; 43(6): 809-12.

7. Cruz GMG, Santana SKAA, Santana JL, Ferreira RMRS; Neves PM, Faria MNZ. Complicações Pós-Operatórias Cirúrgicas da Hemorroidectomia: Revisão de 76 Casos de Complicações. Rev Bras Coloproct 2007; 27(1): 42-57.

8. Fillmann EEP, Fillmann LS, Fillmann HS, Pandolfo G. Antibioticoprofilaxia no Tratamento Cirúrgico da Doença Hemorroidária: Efeito Sobre o Controle da Dor Pós-Operatória , Cicatrização das Feridas e Complicações. Rev Bras Coloproct 2004; 24(1): 5-9.
9. Santos HA. Doença Hemorroidária. In: Cruz GMG. Coloproctologia ii: coloproctologia propedêutica nosológica. Rio de Janeiro: Editora Revinter 1999; 1139-56.

10. Cruz GMG, Ferreira RMRS, Neves PM. Doença Hemorroidária - Aspectos Epidemiológicos e Diagnósticos de 9.289 Pacientes Portadores de Doença Hemorroidária. Rev Bras Coloproct 2006;26(1): 6-23.

11. McConnel JC, Khubchandani IT - Long-term follow-up of closed hemorrhoidectomy. Dis Colon Rectum 1983; 26:797-9.

12. Bleday R, Pena JP, Rothenberger. Symptomatic hemorrhoids: current incidence and complications of operative treatment. Dis Colon Rectum 1992; 35:477-8.

13. Kamphuis ET, Ionescu TI, Kuipers PW. Recovery of storage and emptying functions of the urinary bladder after spinal anesthesia wit lidocaine and with bupivacaine in men. Anesthesiology 1998; 88:310-6.

14. Braum Filho LJ, Casado MRT. Analgesia x efeitos colaterais de doses diferentes de morfina no espaço peridural. Rev Med Aeronaut Bras 1985; 38(1): 345-7.

15. Imbelloni LE, Vieira EM, Gouveia MA, Cordeiro JA. Raquianestesia posterior para cirurgias anorretais em regime ambulatorial. Estudo piloto. Rev Bras Anestesiol 2004;54(6):765-9.

Endereço para correspondência: CARLOS HENRRIQUE MARQUES DOS SANTOS

Rua: Aluisio de Azevedo, 606

Monte Líbano - Campo Grande, MS

CEP- 79004-050

Email: chenrriquems@yahoo.com.br Tel: (67) 8157-7171 